\title{
Degen Generalized Cylinders and Their Properties
}

\author{
Liangliang $\mathrm{CaO}^{1}$, Jianzhuang $\mathrm{Liu}^{1}$, and Xiaoou Tang ${ }^{1,2}$ \\ 1 Department of Information Engineering, The Chinese University of Hong Kong, \\ Hong Kong, China \\ \{llcao, jzliu, xtang\}@ie.cuhk.edu.hk \\ ${ }^{2}$ Microsoft Research Asia, Beijing, China \\ xitang@microsoft.com
}

\begin{abstract}
Generalized cylinder (GC) has played an important role in computer vision since it was introduced in the 1970s. While studying GC models in human visual perception of shapes from contours, Marr assumed that GC's limbs are planar curves. Later, Koenderink and Ponce pointed out that this assumption does not hold in general by giving some examples. In this paper, we show that straight homogeneous generalized cylinders (SHGCs) and tori (a kind of curved GCs) have planar limbs when viewed from points on specific straight lines. This property leads us to the definition and investigation of a new class of GCs, with the help of the surface model proposed by Degen for geometric modeling. We call them Degen generalized cylinders (DGCs), which include SHGCs, tori, quadrics, cyclides, and more other GCs into one model. Our rigorous discussion is based on projective geometry and homogeneous coordinates. We present some invariant properties of DGCs that reveal the relations among the planar limbs, axes, and contours of DGCs. These properties are useful for recovering DGC descriptions from image contours as well as for some other tasks in computer vision.
\end{abstract}

\section{Introduction}

A generalized cylinder (GC) is a solid obtained by sweeping a planar region along an axis. The planar region is called the cross section of the GC and is not necessarily circular or constant. The axis can also be curved in space. This model was at first proposed by Binford in 1971 [1], and has received extensive attention and become popular in computer vision in the past three decades. Because of their ability to represent objects explicitly and their object-centered coordinate frames derivable from image data, GCs have been applied to shape recovery [2], [3], [4], [5], [6], object modelling [7], [8], [9], [10], model-based segmentation and detection [11], [12], modelling tree branches in computer graphics [13], and designing robot vision systems [14].

From previous work on the study of the properties and recovery of GCs, we can roughly divide GCs into two groups: GC with straight axes and GCs with curved axes. In what follows, we call them straight GCs and curved GCs, respectively. Most of the work considers GCs in single views. Straight homogeneous generalized cylinders (SHGCs) are the most important subset of straight GCs, whose sweeping axes are straight and whose cross sections are scaled along the axes. SHGCs were first defined by Shafer and Kanade [15], and then studied extensively by many researchers [2], [4], [6], [11], [12], [16], [17], [18]. 
Compared with SHGCs, less work on curved GCs has been done. The difficulty is mainly due to two facts: the projection of the axis of a curved GC may not be necessarily the axis of its 2D contours [19], and the angle between the axis and the cross section in the image no longer keeps constant [20]. To interpolate the axis of a curved GC in scattered data, Shani and Ballard proposed an iterative solution of minimizing the torsion of the axis [10]. In [5], Sayd et al. presented a scheme to recover a constrained subset of curved GCs with circular and constant cross sections. Ulupinar and Nevatia focused on a subset of GCs whose axes are planar curves 1 and normal to the constant cross sections [21]. Zerroug and Nevatia studied the invariants and quasi-invariants of a subset of GCs with planar curved axes and with circular (not necessarily constant) cross sections [22]. In [9], Gross considered GCs with planar curved axes or with circular cross sections, and presented an algorithm to recover the GCs using image contours and reflectance information.

The analysis of the previous work on SHGCs and curved GCs is explicitly separate, focusing on special classes of GCs. In this paper, starting from the discussion of the conditions when SHGCs and tori (a kind of curved GCs) have planar limbs, we define and study a new class of GCs, with the help of the surface model proposed by Degen for geometric modeling [23], [24]. We call them Degen generalized cylinders (DGCs), which include SHGCs, tori, quadrics, cyclides, and more other GCs into one model. Our rigorous discussion is based on projective geometry and homogeneous coordinates. We present some invariant properties of DGCs that reveal the relations among the planar limbs, axes, and contours of DGCs. We also discuss how the proposed properties can be used for recovering DGC descriptions from image contours, and for generating good initializations for a new 3D deformable DGC model in 3D data fitting and segmentation.

\section{Planar Limbs and View Directions}

This section discusses two classes of GCs that have planar limbs when viewed from specific directions. These GCs with the property of planar limbs are the motivation of our work.

In this paper, image contours are referred to as the projections of contour generators that are curves in space. There are two kinds of contour generators: limbs and edges [6]. Limb points are the points where the surface turns smoothly away from the observer, and edge points are those where the surface orientation is discontinuous. A limb is sometimes called a rim [25], viewpoint-dependent edge, or virtual edge [26].

Although a curve in 3D space can be formed freely, its projected contours cannot keep all the information of its 3D shape. Fig. 1 shows such a limitation. From the projection of a curve, one cannot judge whether it is planar or not in 3D space. To guess the ability of human vision on recovering 3D information from contours, Stevens assumed that one tends to interpret the 2D projection of a space curve as the projection of a planar curve [27], [28]. We can see this tendency from the projections in Fig. 1 if the space curve is not shown. In differential geometry, the torsion of a planar curve is zero, which was used by Shani and Ballard as the minimization criterion to recover $3 \mathrm{D}$ curved axes [10].

\footnotetext{
${ }^{1}$ A planar curve is a curve lying on a plane in space.
} 


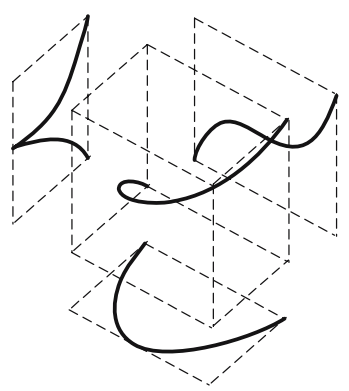

Fig. 1. 2D Projections unable to fully describe the 3D information of the space curve

Marr also assumed that limbs are planar in human visual interpretation. With this assumption and other constraints, Marr showed that human beings always interpret the projected surface as part of a GC; limbs being planar is a basic assumption in the study of reconstructing object surfaces in Marr's fundamental vision theory [25].

However, this assumption does not hold generally as pointed out by Koenderink [29]. He showed that the contour of a torus, which is a curved GC, is often the projection of a non-planar limb. Later Ponce and Chelberg revealed that even SHGCs cannot possess planar limbs from all viewing directions [16]. Fig. 2 gives such an example, where the bold black curves are the intersection of a plane and the GC's surface. From the two viewing directions, the limbs in Fig. 2(a) are planar, but the limbs in Fig. 2(b) are not. Now we discuss in what conditions SHGCs and tori can have planar limbs.

We use the similar notation and the coordinate system as those in [6] and [16]. Suppose that the axis of a SHGC coincides with the $z$-axis as shown in Fig. 3. The surface of a SHGC can be represented in the polar coordinate system by

$$
\mathbf{x}(z, \theta)=\rho(\theta) r(z) \cos \theta \mathbf{i}+\rho(\theta) r(z) \sin \theta \mathbf{j}+z \mathbf{k}
$$

where $z \in[a, b], \theta \in[0,2 \pi]$, and $\rho$ defines the reference cross section on the $x$ - $y$ plane, and $r$ defines the scaling sweeping rule of the SHGC. Let $\mathbf{v}$ be the viewing direction, and $\mathbf{n}$ be the normal vector to the surface at the points on a limb. Then according to the definition of limbs, we have the relation

$$
\mathbf{v} \cdot \mathbf{n}=0
$$

Proposition 1. A SHGC has planar limbs when the viewing direction is normal to the axis of the SHGC under orthographic projection.

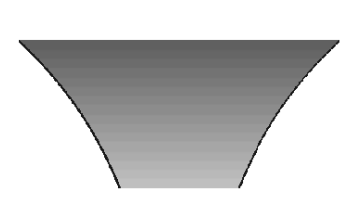

(a)

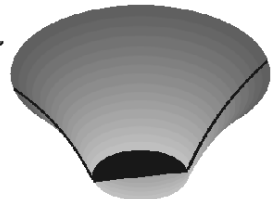

(b)

Fig. 2. (a) Planar limbs. (b) Non-planar limbs 
Proof. Assume the viewing direction is given by its spherical coordinate $(\alpha, \beta)$ (see Fig. 3). Then

$$
\mathbf{v}=\sin \beta \cos \alpha \mathbf{i}+\sin \beta \sin \alpha \mathbf{j}+\cos \beta \mathbf{k} .
$$

With (2), Ponce [6] proved that points on a limb satisfy

$$
\rho^{2} r^{\prime} \cos \beta=\left[\rho(\theta) \cos (\theta-\alpha)+\rho^{\prime}(\theta) \sin (\theta-\alpha)\right] \sin \beta .
$$

When $\mathbf{v}$ is normal to $\mathbf{k}, \beta=90^{\circ}$. Hence

$$
\rho(\theta) \cos (\theta-\alpha)+\rho^{\prime}(\theta) \sin (\theta-\alpha)=0,
$$

which implies a function $\theta$ of $\alpha$ only (independent of $z$ ), i.e., $\theta=f(\alpha)$. We can write the limb equation as

$$
\begin{aligned}
\mathbf{l}(z) & =\mathbf{x}(z, f(\alpha)) \\
& =r(z) \rho(f(\alpha))(\cos f(\alpha) \mathbf{i}+\sin f(\alpha) \mathbf{j})+z \mathbf{k} \\
& =r(z) \mathbf{u}(\alpha)+z \mathbf{k},
\end{aligned}
$$

where $\mathbf{u}(\alpha)=\rho(f(\alpha))(\cos f(\alpha) \mathbf{i}+\sin f(\alpha) \mathbf{j})$. From $[6$,

$$
\begin{aligned}
& \mathbf{l}^{\prime \prime}(z)=r^{\prime \prime}(z) \mathbf{v}(\alpha) \\
& \mathbf{l}^{\prime \prime \prime}(z)=r^{\prime \prime \prime}(z) \mathbf{v}(\alpha) \\
& \mathbf{l}^{\prime \prime}(z) \times \mathbf{l}^{\prime \prime \prime}(z)=\mathbf{0}
\end{aligned}
$$

Hence $\mathbf{l}^{\prime}(z) \times \mathbf{l}^{\prime \prime}(z) \times \mathbf{l}^{\prime \prime \prime}(z)=\mathbf{0}$, which indicates that the limb is a planar curve, because the torsion of a planar curve is equal to zero [30].

Although a torus (a curved GC) does not belong to the class of SHGCs, it also has planar limbs when viewed from specific directions. Note that the axis of a torus is a circle inside the torus.

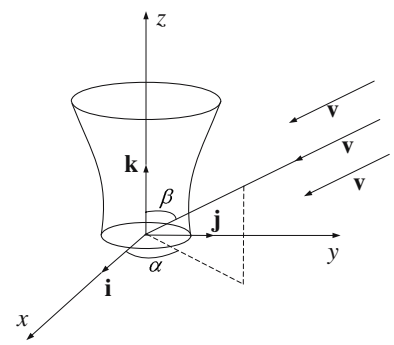

Fig. 3. The coordinate system with a SHGC and the viewing direction $\mathbf{v}$

Proposition 2. A torus has planar limbs while viewed from a point where the line through the point and the torus center is orthogonal to the torus axis. 


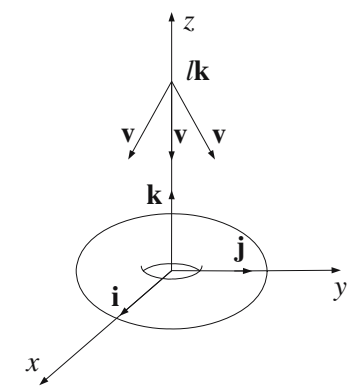

Fig. 4. A torus with the viewpoint at the $z$-axis

Proof. Without loss of generality, we assume that the axis of the torus is located on the $x-y$ plane, the center of it coincides with the origin, and the viewpoint is at $l \mathbf{k}$, as shown in Fig. 4 Then the surface of the torus can be parameterized by [31]

$$
\mathbf{x}(z, \theta)=(R-r \cos z) \cos \theta \mathbf{i}+(R-r \cos z) \sin \theta \mathbf{j}+r \sin z \mathbf{k} .
$$

The normal to the surface is given by

$$
\begin{aligned}
\mathbf{n}(z, \theta)= & \frac{\partial \mathbf{x}}{\partial z} \times \frac{\partial \mathbf{x}}{\partial \theta} \\
= & -r \cos z(R-r \cos z) \cos \theta \mathbf{i}-r \cos z(R-r \cos z) \sin \theta \mathbf{j} \\
& +(R+r \sin z)(R-r \cos z) \mathbf{k} .
\end{aligned}
$$

The viewing direction from $l \mathbf{k}$ to the surface is

$$
\mathbf{v}(z, \theta)=\mathbf{x}(z, \theta)-l \mathbf{k} .
$$

Substituting $\mathbf{v}$ in (12) and $\mathbf{n}$ in (11) into (2) yields

$$
r l \sin z+R r \cos z-R r \sin z-r^{2}+R l=0,
$$

which implies a function $z$ of $l$ only (independent of $\theta$ ). i.e., $z=g(l)$. Thus we can write the limb equation as

$$
\mathbf{l}(\theta)=\mathbf{x}(g(l), \theta)=(R-r \cos g(l))(\cos \theta \mathbf{i}+\sin \theta \mathbf{j})+r \sin g(l) \mathbf{k} .
$$

It is easy to show

$$
\mathbf{l}^{\prime}(\theta) \times \mathbf{l}^{\prime \prime}(\theta) \times \mathbf{l}^{\prime \prime \prime}(\theta)=\mathbf{0} .
$$

Thus the limb is planar since its torsion is zero.

\section{DGCs in Homogeneous Coordinates}

We have shown that SHGCs and tori have planar limbs when viewed from some specific directions. There are also other curved GCs sharing the same property. This property leads us to the investigation of DGCs. For mathematical convenience, we will mainly use homogeneous coordinates and projective geometry in the following discussion of DGCs. 


\subsection{Homogeneous Coordinates}

Homogeneous coordinates are used in projective geometry [32]. They are a useful tool in computer vision and graphics. Points in homogeneous coordinates are represented by vectors $\mathbf{p}=(w, x, y, z)^{T} \in \mathbb{R}^{4} \backslash\left\{(0,0,0,0)^{T}\right\}$. The $w, x, y, z$ are called homogeneous coordinates of p. p and $\rho \mathbf{p}$ with $\rho \in \mathbb{R} \backslash\{0\}$ define the same point. Given a point $\mathbf{p}=(w, x, y, z)^{T}$ with $w \neq 0$ in homogeneous coordinates, its corresponding point $\overline{\mathbf{p}}$ in Cartesian coordinates is

$$
\overline{\mathbf{p}}=(\bar{x}, \bar{y}, \bar{z})^{T}=\left(\frac{x}{w}, \frac{y}{w}, \frac{z}{w}\right)^{T} .
$$

If $w=0$, the point $(0, x, y, z)$ stands for a point at infinity (called an ideal point).

Orthogonal projection can be treated as a special case of perspective projection when the viewpoint is at infinity. Thus under perspective projection, Proposition 1 states that a SHGC has planar limbs when the viewpoint is at infinity and the viewing direction is normal to the axis of the SHGC.

Using homogeneous coordinates, points on a straight line $\mathbf{L}$ can be represented by

$$
\mathbf{L}=\alpha \mathbf{a}+\beta \mathbf{b},
$$

where $\alpha, \beta \in \mathbb{R}$ and $\mathbf{a}, \mathbf{b}$ are two independent points in the projective space. In what follows, we denote the line $\mathbf{L}$ by $\mathbf{a} \wedge \mathbf{b}$. Similarly, points on a plane $\mathbf{P}$ can be represented by

$$
\mathbf{P}=\alpha \mathbf{a}+\beta \mathbf{b}+\gamma \mathbf{c}
$$

where $\alpha, \beta, \gamma \in \mathbb{R}$ and $\mathbf{a}, \mathbf{b}, \mathbf{c}$ are three independent points. We denote the plane $\mathbf{P}$ by $\mathbf{a} \wedge \mathbf{b} \wedge \mathbf{c}$. Therefore, a curve $\mathbf{C}(s)$ is planar if it can be written in this form

$$
\mathbf{C}(s)=p_{1}(s) \mathbf{a}+p_{2}(s) \mathbf{b}+p_{3}(s) \mathbf{c} .
$$

To verify whether a curve is planar or not, this way is more convenient than calculating the torsion of the curve in Cartesian coordinates.

\subsection{Degen Surfaces}

Degen proposed a novel surface model for geometric modelling in [23] and [24]. We call those surfaces Degen surfaces. They cover a wide range of curved surfaces such as those showed in Fig. [5. A Degen surface is parameterized by the following equation in homogeneous coordinates

$$
\mathbf{X}(u, v)=\alpha(u) \mathbf{a}+\beta(u) \mathbf{b}+\gamma(v) \mathbf{c}+\delta(v) \mathbf{d}=\mathbf{p}(u)+\mathbf{q}(v),
$$

where $\mathbf{p}(u)=\alpha(u) \mathbf{a}+\beta(u) \mathbf{b}, \mathbf{q}(v)=\gamma(v) \mathbf{c}+\delta(v) \mathbf{d}, u \in\left[u_{1}, u_{2}\right], v \in\left[v_{1}, v_{2}\right]$, $\mathbf{a}, \mathbf{b}, \mathbf{c}, \mathbf{d}$ are independent, and $\alpha, \beta, \gamma, \delta$ are certain functions. The two straight lines $\mathbf{a} \wedge \mathbf{b}$ and $\mathbf{c} \wedge \mathbf{d}$ are called the axes of the Degen surface. 


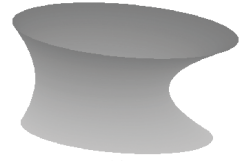

(a)

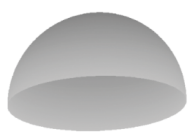

(d)

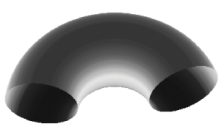

(b)

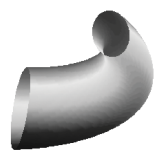

(e)

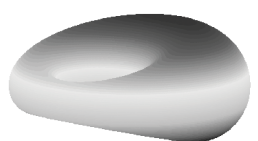

(c)

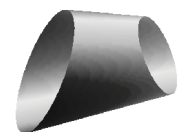

(f)

Fig. 5. Some examples of Degen Surfaces including SHGCs(a), an open torus(b), a cyclide(c), a quadric(d), and more other GCs (e,f), respectively

\subsection{DGCs}

Before defining DGCs, we show that SHGCs and tori can be represented in the form of Degen surfaces in homogeneous coordinates. The parameterized surface of a SHGC in homogeneous coordinates is simply

$$
\mathbf{X}(u, v)=(1, \rho(u) r(v) \cos u, \rho(u) r(v) \sin u, v)^{T},
$$

where the $z$ and $\theta$ in (1) are replaced by $u$ and $v$, respectively. Then $\mathbf{X}(u, v)=\mathbf{p}(u)+$ $\mathbf{q}(v)$ with

$$
\begin{aligned}
& \mathbf{p}(u)=(0, \rho(u) \cos u, \rho(u) \sin u, 0)^{T} \\
& \mathbf{q}(v)=\frac{1}{r(v)}(1,0,0, v)^{T} .
\end{aligned}
$$

Furthermore

$$
\begin{aligned}
& \mathbf{p}(u)=\rho(u)(\cos u) \mathbf{a}+\rho(u)(\sin u) \mathbf{b} \\
& \mathbf{q}(v)=\frac{1}{r(v)} \mathbf{c}+\frac{v}{r(v)} \mathbf{d}
\end{aligned}
$$

with $\mathbf{a}=(0,1,0,0)^{T}, \mathbf{b}=(0,0,1,0)^{T}, \mathbf{c}=(1,0,0,0)^{T}, \mathbf{d}=(0,0,0,1)^{T}$.

Similarly, replacing the $z$ and $\theta$ in (10) with $u$ and $v$, respectively, we can show that a torus belongs to a Degen surface by

$$
\begin{aligned}
& \mathbf{p}(u)=\frac{1}{R-r \cos u}(1 / r, 0,0, \sin u)^{T}=\frac{1}{r(R-r \cos u)} \mathbf{a}+\frac{\sin u}{R-r \cos u} \mathbf{b} \\
& \mathbf{q}(v)=\frac{1}{r}(0, \cos v, \sin v, 0)^{T}=\frac{\cos v}{r} \mathbf{c}+\frac{\sin v}{r} \mathbf{d},
\end{aligned}
$$

with $\mathbf{a}=(1,0,0,0)^{T}, \mathbf{b}=(0,0,0,1)^{T}, \mathbf{c}=(0,1,0,0)^{T}, \mathbf{d}=(0,0,1,0)^{T}$.

Definition 1. On a Degen surface with the parametrization of $\mathbf{X}(u, v)$, when $v=v_{0}$ is fixed, the curve $\mathbf{C}_{1}(u)=\mathbf{X}\left(u, v_{0}\right)$ is called a u-curve; when $u=u_{0}$ is fixed, the curve $\mathbf{C}_{2}(v)=\mathbf{X}\left(u_{0}, v\right)$ is called a v-curve. 
In the above examples, the $u$-curves of a SHGC are $(0, \rho(u) \cos u, \rho(u) \sin u, 0)^{T}+$ $\mathbf{q}\left(v_{0}\right)$, which are closed when $u \in[0,2 \pi]$; the $v$-curves of the SHGC are $\mathbf{p}\left(u_{0}\right)+$ $\frac{1}{r(v)}(1,0,0, v)^{T}$. Both the $u$-curves and $v$-curves of a torus are circles, which are also closed.

On a Degen surface with $u \in\left[u_{1}, u_{2}\right], v \in\left[v_{1}, v_{2}\right]$, the family of $u$-curves $\left\{\mathbf{C}_{1}(u)=\right.$ $\left.\mathbf{X}\left(u, v_{0}\right) \mid v_{0} \in\left[v_{1}, v_{2}\right]\right\}$ covers the whole surface. Thus a Degen surface can be seen as a surface obtained by sweeping a $u$-curve when $v_{0}$ varies from $v_{1}$ to $v_{2}$. If the $u$-curve is closed, the region bounded by it can be regarded as the cross section of a GC. Note that all the $u$-curves and $v$-curves are planar as stated in Lemma 1 in Section 4.

Definition 2. A Degen generalized cylinder $(D G C)$ is a solid bounded by a Degen surface $\mathbf{X}(u, v)=\alpha(u) \mathbf{a}+\beta(u) \mathbf{b}+\gamma(v) \mathbf{c}+\delta(v) \mathbf{d}$ with closed $u$-curves, or closed $v$-curves, or both. The axes of the DGC are the two straight lines $\mathbf{a} \wedge \mathbf{b}$ and $\mathbf{c} \wedge \mathbf{d}$.

Obviously, the surface of a DGC is a Degen surface. However, a Degen surface with neither $u$-curves nor $v$-curves closed does not form a DGC. Fig. 6 gives such an example. The Degen surfaces showed in Fig. 5 form six DGCs if the cross sections are considered as regions instead of curves.

It should be emphasized that a conventional GC has only one axis and the axis of a conventional curved GC is a curve. It is often more difficult to recover curved axes than to recover straight axes.

\section{Properties of DGCs}

In this section, we present the properties of DGCs that are useful for some computer vision tasks.

Proposition 3. The axis of a SHGC coincides with one of the two axes of the DGC that is the corresponding representation of the SHGC in homogeneous coordinates. Another axis of the DGC is a line at infinity.

Proof. When a SHGC is written as (1), its axis is the $z$-axis (Fig. 3). The same SHGC can be represented in the form of a DGC as in (21)-(25). One axis of the DGC is $\mathbf{c} \wedge \mathbf{d}$, i.e., a line passing through $(1,0,0,0)^{T}$ and $(0,0,0,1)^{T}$, which denotes the $z$-axis in homogeneous coordinates. Another axis of the DGC is a line at infinity, which passes through the two ideal points $\mathbf{a}=(0,1,0,0)^{T}$ and $\mathbf{b}=(0,1,0,0)^{T}$.

It is also easy to find the two axes of a torus when it is represented in the form of a DGC. Suppose a torus in Euclidean geometry is expressed by (10). From (26) and 27, we

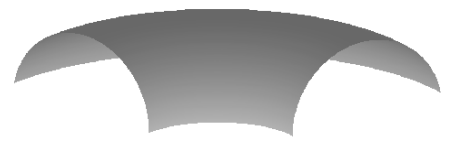

Fig. 6. A Degen surface with neither $u$-curves nor $v$-curves closed 
see that one axis of the torus is $\mathbf{a} \wedge \mathbf{b}$ with $\mathbf{a}=(1,0,0,0)^{T}$ and $\mathbf{b}=(0,0,0,1)^{T}$, which is the $z$-axis in homogeneous coordinates. Another axis is $\mathbf{c} \wedge \mathbf{d}$ with $\mathbf{c}=(0,1,0,0)^{T}$ and $\mathbf{d}=(0,0,1,0)^{T}$, which is a line through the two ideal points $\mathbf{c}$ and $\mathbf{d}$ at infinity.

As pointed out in Propositions 1 and 2, both SHGCs and tori have planar limbs when viewed from the special directions. Now we show that all DGCs have this property. At first, we give two lemmas that are proved in [23].

Lemma 1. All the u-curves and v-curves of a DGC are planar.

Lemma 2. All the tangent planes on a u-curve $\mathbf{X}\left(u, v_{0}\right)$ (v-curve $\mathbf{X}\left(u_{0}, v\right)$, respectively) pass through the same point $\gamma^{\prime}\left(v_{0}\right) \mathbf{c}+\delta^{\prime}\left(v_{0}\right) \mathbf{d}\left(\alpha^{\prime}\left(u_{0}\right) \mathbf{a}+\beta^{\prime}\left(u_{0}\right) \mathbf{b}\right.$, respectively).

Proposition 4. A DGC has planar limbs when viewed from points on its two axes $\mathbf{a} \wedge \mathbf{b}$ and $\mathbf{c} \wedge \mathbf{d}$, and the planar limbs are $u$-curves and $v$-curves.

Proof. From Lemma2 we know that all the tangent planes on a $u$-curve $X\left(u, v_{0}\right)$ pass through the point $\gamma^{\prime}\left(v_{0}\right) \mathbf{c}+\delta^{\prime}\left(v_{0}\right) \mathbf{d}$. All such points with different values of $v_{0}$ lie on the axis $\mathbf{c} \wedge \mathbf{d}$. Therefore, if the DGC is observed from one of the points, the viewing directions must lie on these tangent planes at points on the $u$-curves. Thus the $u$-curve becomes a limb of the DGC. By Lemma 1 the limb is planar. Similarly, the DGC has planar limbs when observed from points on another axis $\mathbf{a} \wedge \mathbf{b}$.

Proposition 5. For any two contour points from the same u-curve (v-curve, respectively), the tangents to the contours at the two points intersect on the projection of the axis $\mathbf{c} \wedge \mathbf{d}(\mathbf{a} \wedge \mathbf{b}$, respectively).

Proof. From Lemma 2, all the tangent planes of the $u$-curve ( $v$-curve, respectively) meet at the same point on the axis $\mathbf{c} \wedge \mathbf{d}(\mathbf{a} \wedge \mathbf{b}$, respectively). Since the tangent plane at a point of the limb is projected onto the tangent at the corresponding point on the contour generated by the limb [33], this proposition holds.

Fig. 7 illustrates this invariant property. Note that when a DGC is a SHGC, one axis becomes the axis of the SHGC (Proposition 3). Thus the SHGC's invariant property stated in Lemma 4 in Ponce et al.'s work [6] becomes a special case of Proposition5]

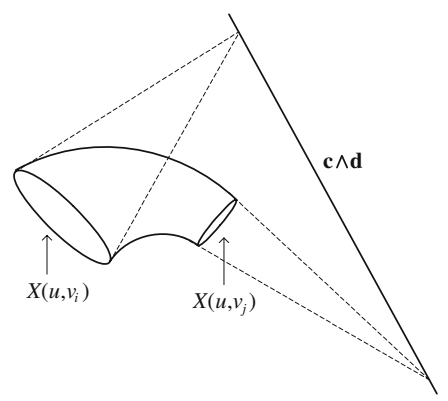

Fig. 7. Illustration of Proposition 5 


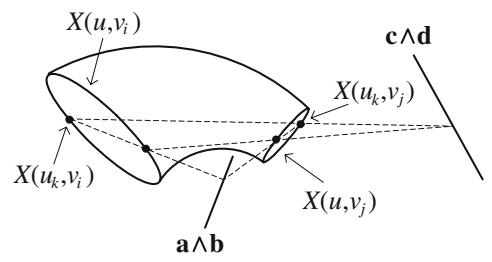

Fig. 8. Illustration of Proposition 6

Definition 3. Let $\mathbf{X}\left(u, v_{i}\right)$ and $\mathbf{X}\left(u, v_{j}\right)$ be two u-curves of a DGC. Two points $\mathbf{X}\left(u_{k}, v_{i}\right)$ and $\mathbf{X}\left(u_{k}, v_{j}\right)$ on the two u-curves define a line of correspondence from the two u-curves. Let $\mathbf{X}\left(u_{m}, v\right)$ and $\mathbf{X}\left(u_{n}, v\right)$ be two $v$-curves of a DGC. Two points $\mathbf{X}\left(u_{m}, v_{q}\right)$ and $\mathbf{X}\left(u_{n}, v_{q}\right)$ on the two $v$-curves define a line of correspondence from the two v-curves.

Proposition 6. All the lines of correspondence from any two u-curves ( $v$-curves, respectively) of a DGC intersect at the same point on the axis $\mathbf{c} \wedge \mathbf{d}(\mathbf{a} \wedge \mathbf{b}$, respectively).

Proof. Let $\mathbf{X}\left(u, v_{i}\right)$ and $\mathbf{X}\left(u, v_{j}\right)$ be two $u$-curves as shown in Fig. 8, the line of correspondence passing through the two points $\mathbf{X}\left(u_{k}, v_{i}\right)$ and $\mathbf{X}\left(u_{k}, v_{j}\right)$ can be expressed as

$$
\mathbf{X}\left(u_{k}, v_{i}\right)+\lambda \mathbf{X}\left(u_{k}, v_{j}\right), \quad \lambda \in \mathbb{R} .
$$

When $\lambda=-1$,

$$
\begin{aligned}
\mathbf{X}\left(u_{k}, v_{i}\right)-\mathbf{X}\left(u_{k}, v_{j}\right) & =\left[\mathbf{p}\left(u_{k}\right)+\mathbf{q}\left(v_{i}\right)\right]-\left[\mathbf{p}\left(u_{k}\right)+\mathbf{q}\left(v_{j}\right)\right] \\
& =\mathbf{q}\left(v_{i}\right)-\mathbf{q}\left(v_{j}\right) \\
& =\left[\gamma\left(v_{i}\right)-\gamma\left(v_{j}\right)\right] \mathbf{c}+\left[\delta\left(v_{i}\right)-\delta\left(v_{j}\right)\right] \mathbf{d},
\end{aligned}
$$

which is a point on the axis $\mathbf{c} \wedge \mathbf{d}$. Since this point is independent of $u_{k}$, all such lines from the two $u$-curves intersect at this point. In the same way, we can also prove that the proposition is true for the lines of correspondence from two $v$-curves.

From Proposition 6, we can obtain a corollary, the geometry of which is illustrated in Fig. 9. The proof is omitted due to space limitation.

Corollary 1. In the general case, the two axes $\mathbf{a} \wedge \mathbf{b}$ and $\mathbf{c} \wedge \mathbf{d}$ of a DGC can be determined from a pair of $u$-curves and a pair of $v$-curves of the DGC.

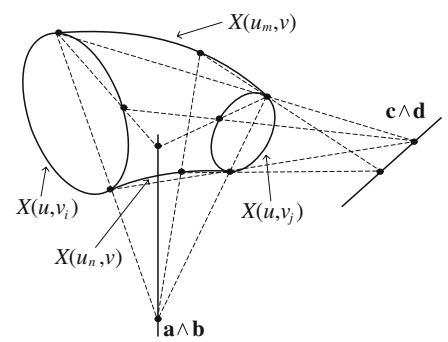

Fig. 9. Illustration of Corollary 1 


\section{Conclusions}

GCs have been used in many applications of computer vision. Previous work on GCs focuses on relatively narrow sets of GCs. In this paper, we have proposed a new set of GCs, called Degen generalized cylinders (DGCs). DGCs cover a wide range of GCs, including SHGCs, tori, quadrics, cyclides, and more other GCs into one unified model. We have presented a number of properties existing in DGCs. Our rigorous discussion is based on homogeneous coordinates in projective geometry, which is more general than Euclidean geometry. The invariant properties of DGCs reveal the relations among the planar limbs, axes, and contours of DGCs. These properties can be used for recovering DGC descriptions from image contours, representing GCs in computer vision and graphics, and modeling surface warping in 3D animation.

\section{Acknowledgments}

The authors would like to thank the anonymous reviewers for their useful comments. This work is supported by RGC Research Direct Grant 2050369, CUHK.

\section{References}

1. Binford, T.: Visual perception by computer. IEEE Conf. Systems and Control (1971)

2. Sato, H., Binford, T.: Finding and recovering SHGC objects in an edge image. CVGIP: Graphical Model and Image Processing 57(3) (1993) 346-358

3. Dhome, M., Glachet, R., Lapreste, J.: Recovering the scaling function of a SHGC from a single perspective view. IEEE Proc. Computer Vision and Pattern Recognition (1992) 36-41

4. Ulupinar, F., Nevatia, R.: Shape from contour: Straight homogeneous generalized cylinders and constant cross-section generalized cylinders. IEEE Trans. Pattern Analysis and Machine Intelligence 17(2) (1995) 120-135

5. Sayd, P. Dhome, M., Lavest, J.: Recovering generalized cylinders by monocular vision. Object Representation in Computer Vision II (1996) 25-51

6. Ponce, J., Chelberg, D., Mann, W.: Invariant properties of straight homogeneous generalized cylinders and their contours. IEEE Trans. Pattern Analysis and Machine Intelligence 11(9) (1989) 951-966

7. O'Donnell, T. Boult, T., Fang, X., Gupta, A.: The extruded generalized cylinder: A deformable model for object recovery. Proc. IEEE Conf. Computer Vision and Pattern Recognition (1994) $174-181$

8. O’Donnell, T., Dubuisson-Jolly, M.P., Gupta, A.: A cooperative framework for segmentation using 2-D active contours and 3-D hybrid models as applied to branching cylindrical structures. Proc. Int'l Conf. Computer Vision (1998) 454 - 459

9. Gross, A.D.: Analyzing generalized tubes. Proc. SPIE Conf. Intelligent Robots and Computer Vision XIII: 3D Vision, Product 2354 (1994)

10. Shani, U., Ballard, D.H.: Splines as embeddings for generalized cylinders. Computer Vision, Graphics, and Image Processing (CVGIP) 27(2) (1984) 129-156

11. Zerroug, M., Nevatia, R.: Segmentation and recovery of SHGCS from a real intensity image. European Conf. Computer Vision (1994) 319-330

12. Gross, A.D., Boult, T.E.: Recovery of SHGCs from a single intensity view. IEEE Trans. Pattern Analysis and Machine Intelligence 18(2) (1996) 161-180 
13. Bloomenthal, J.: Modeling the mighty maple. Proc. SIGGRAPH '85 19(3) (1985)

14. Brooks, R. A., R.G., Binford, T.O.: The ACRONYM model-based vision system. Proc. of 6th Int'1 Joint Conf. Artificial Intelligence (1979) 105-113

15. Shafer, S., Kanade, T.: The theory of straight homogeneous generalized cylinders and a taxonomy of generalized cylinders. Technical Report, Carnegie Mellon Universit (1983)

16. Ponce, J., Chelberg, D.: Finding the limbs and cusps of generalized cylinders. Int'l Journal of Computer Vision 1 (1987) 195-210

17. Ulupinar, F., Nevatia, R.: Perception of 3-D surfaces from 2-D contours. IEEE Trans. Pattern Analysis and Machine Intelligence 15(1) (1993) 3-18

18. Ulupinar, F., Nevatia, R.: Recovery of 3-D objects with multiple curved surfaces from 2-D contours. Artificial Intelligence 67(1) (1994) 1-28

19. P.J.Giblin, B.B.Kimia: Transitions of the 3D medial axis under a one-parameter family of deformations. European Conf. Computer Vision (2002) 718-724

20. Zerroug, M., Nevatia, R.: Three-dimensional descriptions based on the analysis of the invariant and quasi-invariant properties of some curved-axis generalized cylinders. IEEE Trans. Pattern Analysis and Machine Intelligence 18(3) (1996) 237-253

21. Ulupinar, F., Nevatia, R.: Recovering shape from contour for constant cross section generalized cylinders. Proc. IEEE Conf. Computer Vision and Pattern Recognition (1991) 674-676

22. Zerroug, M., Nevatia, R.: Quasi-invariant properties and 3-D shape recovery of non-straight, non-constant generalized cylinders. Proc. IEEE Conf. Computer Vision and Pattern Recognition (1993) 96-103

23. Degen, W.L.F.: Nets with plane silhouettes. Proc. IMA Conf. the Mathematics of Surfaces V (1994) 117-133

24. Degen, W.L.F.: Conjugate silhouette nets. Curve and surface design: 99 (2000) 37-44

25. Marr, D.: Vision: A Computational Investigation into the Human Representation and Processing of Visual Information. Henry Holt Company (1982)

26. Zhu, Q.: Virtual edges, viewing faces, and boundary traversal in line drawing representation of objects with curved surfaces. Int'l J. Computers and Graphics 15(2) (1991) 161-173

27. Stevens, K.: The visual interpretation of surface contours. Artificial Intelligence 17(1-3) (1981) 47-73

28. Stevens, K.: Implementation of a theory for inferring surface shape from contours. MIT AI Memo-676 (1982)

29. Koenderink, J.: What does the occluding contour tell us about solid shape? Perception 13 (1984) 321-330

30. Carmo, D.: Differential geometry of curves and surfaces. Englewood Cliffs, N.J. : Prentice Hal (1976)

31. Marsh, D.: Applied Geometry for Computer Graphics and CAD. Springer Undergraduate Mathematics Series. Springer (1999)

32. E.A.Maxwell: General Homogeneous Coordinates in Space of Three Dimensions. Cambridge University Press (1951)

33. Ulupinar, F., Nevatia, R.: Shape from contour: Straight homogeneous generalized cones. Proc. Int'l Conf. Pattern Recognition (1990) 582-586 